

\title{
Synthesis and Anticoccidial Activities of Quinoline Carboxylate Derivatives with Methyl (E)-2-(3-methoxy)acrylate Moiety
}

Yuan Liu, Ya-Biao Weng, Zhao-Bing Chen and Yu-Liang Wang*

Faculty of Chemistry, Sichuan University, Chengdu 610065, P.R. China

*Corresponding author: Tel: +86 15378195811; E-mail: chemwang2008@163.com; luobinhxscu@126.com

A series of novel quinoline carboxylate derivatives with methyl (E)-2-(3-methoxy) acrylate group were designed and synthesized as anticoccidial medicines. The structures were confirmed by ${ }^{1} \mathrm{H}$ NMR, IR and HR-MS spectra. The biological activities were primarily evaluated according to the anticoccidial index method. The results indicated that these compounds (7c, 7d, 7e, $7 \mathbf{g})$ exhibited anticoccidial activities against Eimeria tenella. In particular, the anticoccidial index of 6-decyloxy-7-ethoxy-4-\{6-[2-(2-methoxy-1-methoxycarbonyl-vinyl)phenoxy]pyrimidin-4-yloxy $\}$ quinoline-3-carboxylic acid ethyl ester (7e) was 168.7, which indicated that the compound has a good anticoccidial activity.

Key Words: Quinoline carboxylate derivatives, Methyl (E)-2-(3-methoxy)acrylate, Anticoccidial activity, Identifition, Synthesis. |

\section{INTRODUCTION}

Coccidiosis is one of the most prevalent diseases in world's poultry industry that causes poor growth rate, high morbidity and high mortality rates ${ }^{1,2}$. It is an infection of intestinal epithelium caused by protozoan parasite of the genus Eimeria ${ }^{3,}$ ${ }^{4}$. Anticoccidial agents are extensively used to control coccidiosis, but the coccidia was very easy to develop resistance to all of the drugs that have been introduced ${ }^{5,6}$. Since there are no ways to restrain drug-resistance at present, the research work on new anticoccidial drugs should be continued.

Quinoline carboxylate is a kind of potent anticoccidial active substance against protozoan parasite genus Eimeria ${ }^{7}$. But the anticoccidial effects of quinoline carboxylate had declined in the poultry industry due to the development of drug resistance ${ }^{8}$. In this article quinoline carboxylate moiety, which was the key factor for the biological activity, was reserved and $\beta$-methoxy acrylic acid was introduced to synthesize new derivatives. The structure of the new compounds is different from traditional quinoline carboxylate, might be sensitive to drug -resistance coccidiosis.

Eight of novel ethyl quinoline carboxylate derivatives with methyl (E)-2-(3-methoxy)acrylate group at C-4 were designed and synthesized. The general synthetic methods of the target compounds (7a-7h) was shown in Scheme-I. The biological activities of these compounds (7a-7h) were primarily evaluated according to the anticoccidial index method ${ }^{9,10}$. These compounds have good anticoccidial activities and can be used as anticoccidial drugs in the fowl.

\section{EXPERIMENTAL}

$N, N$-Dimethyl formamide was dried and distilled over anhydrous magnesium sulfate. Melting points were resolved using XRC-1 melting point apparatus (Sichuan University Instrument Inc., Chengdu, China) without being corrected. Analytical thin-layer chromatography was performed on silica plates GF254, the spots on TLC were visualized with ultraviolet (UV) light. Mass spectra (MS) were obtained with the Agilent 6210 (DOF-MAS) spectrometer (Agilent Inc., Santa Clara, CA, USA) using the electrospray ionization (ESI) method. ${ }^{1} \mathrm{H}$ NMR spectra were performed in $\mathrm{CDCl}_{3}$ solution on a Varian Unity Inova-400 spectrometer (Varian Inc., Palo Alto, CA, USA) with TMS as the internal standard. A PerkinElmer 16PC-FT instrument (Perkin-Elmer Inc., Norwalk Conn, CA, USA) was used to determine the IR spectra. Intermediate 5e was commercially acquirable. Intermediate $\mathbf{1}$ was synthesized according to the literature ${ }^{11}$. Intermediate $\mathbf{5 h}$ was $\mathrm{s}$ ynthesized according to the literature ${ }^{12}$. Intermediates $\mathbf{5 a - 5 d}$ and Intermediates $\mathbf{5 f - 5 g}$ were synthesized according to the literature $^{11}$. Intermediate $\mathbf{6}$ was synthesized according to the literature $^{13}$.

\section{Synthesis}

Synthesis of compound $3 \mathbf{a}^{14}$ : A sample of 4-nitroso-2methylphenol (intermediate 1) $(3.52 \mathrm{~g}, 25.6 \mathrm{mmol})$ was reduced by $10 \% \mathrm{Pd} / \mathrm{C}(0.35 \mathrm{~g})$ catalyzed hydrogenation in ethyl acetate $(40 \mathrm{~mL})$. The reaction was stirred at $40{ }^{\circ} \mathrm{C}$ for $5 \mathrm{~h}$, cooled and directly used to react with diethylethoxymethylene-malonate 
<smiles>CO/C=C(\C(=O)OC)C(=O)OCCc1ccc2c(c1)C(=Cc1ccccc1O)C(=O)O2</smiles><smiles>CCc1ccccc1/C(=C/OC)C(=O)OC</smiles><smiles>[R]c1cc(N=O)ccc1O</smiles><smiles>[R20]c1ccccc1[R]OCCCOC(=O)C(=CNC)C(=O)OCC</smiles><smiles>CCOC(=O)C(=CNC)C(=O)OCC</smiles><smiles>[R20]Oc1cc2c(Oc3cc(Oc4ccccc4/C(=C/OC)C(=O)OC)ncn3)c(C(=O)OC)cnc2cc1[R]</smiles>

7

Reaction reagents and conditions: a. $10 \% \mathrm{Pd} / \mathrm{C}, \mathrm{H}_{2} ;$ b.EMME; c. $\mathrm{R}_{2} \mathrm{Cl}, \mathrm{K}_{2} \mathrm{CO}_{3}, \mathrm{DMF}$ d.diphenyl ether, $250^{\circ} \mathrm{C}$; e. $\mathrm{K}_{2} \mathrm{CO}_{3}, \mathrm{KI}, \mathrm{DMF}$
(EMME; $5.54 \mathrm{~g}, 25.6 \mathrm{mmol}$ ). The mixture was heated under reflux for $2 \mathrm{~h}$. After the mixture was cooled and filtered off, the filtrate was purified through recrystallization in ethanol and dried to form intermediate 3 (yield 72-81\%).

Synthesis of intermediate $4 \mathrm{a}-\mathrm{d}$ and intermediate $4 \mathrm{f}-\mathrm{h}$ : A mixture of intermediate $3(0.79 \mathrm{~g}, 2.70 \mathrm{mmol}), \mathrm{K}_{2} \mathrm{CO}_{3}(0.41$ $\mathrm{g}, 2.97 \mathrm{mmol}$ ) and bromoalkane (R-Br, $2.7 \mathrm{mmmol})$ in dry DMF $(5 \mathrm{~mL})$ was stirred at $80^{\circ} \mathrm{C}$ for $2 \mathrm{~h}$. After the mixture was cooled to room temperature, water was added cooled. The resulted solids was filterd off, recrystallized in ethanol and dried to give intermediate 4 (yield 83-92\%).

Synthesis of Intermediates 5a-5d and intermediates $5 \mathbf{f}$ $\mathbf{5 h}^{\mathbf{1 2}}$ : Diphenyl ether $(10 \mathrm{~mL})$ was heated to $250{ }^{\circ} \mathrm{C}$, then intermediate $\mathbf{4}$ was added and the mixture was kept at $250{ }^{\circ} \mathrm{C}$ for 8-12 min and then cooled to room temperature. Petroleum ether $(20 \mathrm{~mL})$ was added to the mixture and the resulted solid was filtrated, washed with petroleum ether and dried. The crude material was recrystallized in DMF and dried to give intermediate 5 (yield 43-49\%).

Synthesis of intermediate $6^{13}$ : 2-[2-(6-Chloro-pyrimidin4-yloxy)-phenyl]-3-methoxy-acrylic acid was prepared according to the literature ${ }^{13}$. In the synthesis of 2-[2-(6-chloropyrimidin-4-yloxy)-phenyl]-3-methoxy-acrylic acid methyl ester, sodium methoxide was freshly synthesized by sodium and methanol. And the crude product was chromatographed on silica gel using petroleum ether/ethyl acetate $(4: 1)$ as eluent to afford a pure product (yield $48 \%$ ). Intermediate 6 is known compound reported in literature ${ }^{13}$ and were identified by melting point test, ${ }^{1} \mathrm{H} \mathrm{NMR}$ and IR, all data was the same with the literature ${ }^{13}$.

Synthesis of compound 7a-7h: A solution of intermediate $6(0.51 \mathrm{~g}, 1.27 \mathrm{mmol})$ in $3 \mathrm{~mL} \mathrm{DMF}$ was added dropwise to a mixture of intermediate $\mathbf{5 a - h}(1.06 \mathrm{mmol}), 0.06 \mathrm{~g} \mathrm{KI}$,
$0.18 \mathrm{~g}$ (1.38 mmol) $\mathrm{K}_{2} \mathrm{CO}_{3}$ and $25 \mathrm{~mL} \mathrm{DMF}$ under stirring at $90^{\circ} \mathrm{C}$. After $10 \mathrm{~h}$ the mixture was cooled to the room temperature and then $30 \mathrm{~mL}$ water was added. The solid was filterd off, the crude product was chromatographed on a silica gel column using petroleum ether/ethyl acetate $(2: 1 \mathrm{v} / \mathrm{v})$ as the mobile phase to give the target molecule (yield 40-45 \%).

4-\{6-[2-(2-Methoxy-1-methoxycarbonyl-vinyl)-phenoxy]pyrimidin-4-yloxy\}-7-methyl-6-tetradecyloxy-quinoline-3carboxylic acid ethyl ester (7a) : Light yellow solid; yield: $43 \%$; m.p. $135-138{ }^{\circ} \mathrm{C} ;{ }^{1} \mathrm{H}$ NMR spectrum (400 MHz; $\mathrm{d}_{1-}$ $\mathrm{CDCl}_{3}$; TMS): $\delta$ (ppm.) $=0.876(3 \mathrm{H}, \mathrm{t}, J=6.8 \mathrm{~Hz}), 1.261$ $(24 \mathrm{H}, \mathrm{m}), 1.834(3 \mathrm{H}, \mathrm{t}, J=6.8 \mathrm{~Hz}), 2.298(3 \mathrm{H}, \mathrm{s}), 3.612(3 \mathrm{H}$, s), $3.774(3 \mathrm{H}, \mathrm{s}), 4.103(2 \mathrm{H}, \mathrm{t}, J=6.8 \mathrm{~Hz}), 4.398(2 \mathrm{H}, \mathrm{q}, J=$ $6.8 \mathrm{~Hz}), 6.957$ (1H, s), 7.22-7.54 (6H, m), $7.820(1 \mathrm{H}, \mathrm{s}), 8.712$ $(1 \mathrm{H}, \mathrm{s}), 8.861(1 \mathrm{H}, \mathrm{s})$.

IR $\left(\mathrm{KBr}, v_{\max }, \mathrm{cm}^{-1}\right): 3054,2925,2853,1730,1618,1583$, 1465, 1376, 1256, 1217, 1132, 1097, 1026, 943, 859, 802, 748, 689, 617, 550. HR-MS (ESI): Calcd. for $\mathrm{C}_{42} \mathrm{H}_{54} \mathrm{~N}_{3} \mathrm{O}_{8}$ $[\mathrm{M}+\mathrm{H}]^{+}:$728.3911. Found: 728.3902 .

6-Hexadecyloxy-4-\{6-[2-(2-methoxy-1-methoxycarbonyl-vinyl)-phenoxy]-pyrimidin-4-yloxy\}-7-methylquinoline-3-carboxylic acid ethyl ester (7b) : Light yellow solid; yield: $40 \%$; m.p. $136-138{ }^{\circ} \mathrm{C} ;{ }^{1} \mathrm{H}$ NMR spectrum (400 $\mathrm{MHz} ; \mathrm{d}_{1}-\mathrm{CDCl}_{3}$; TMS): $\delta$ (ppm.) $=0.871(3 \mathrm{H}, \mathrm{t}, J=6.4 \mathrm{~Hz})$, $1.252(28 \mathrm{H}, \mathrm{m}), 1.833(3 \mathrm{H}, \mathrm{t}, J=6.0 \mathrm{~Hz}), 2.300(3 \mathrm{H}, \mathrm{s}), 3.610$ $(3 \mathrm{H}, \mathrm{s}), 3.776(3 \mathrm{H}, \mathrm{s}), 4.099(2 \mathrm{H}, \mathrm{t}, J=6.8 \mathrm{~Hz}), 4.402(2 \mathrm{H}, \mathrm{q}$, $J=6.8 \mathrm{~Hz}), 6.983(1 \mathrm{H}, \mathrm{s}) 7.22-7.52(6 \mathrm{H}, \mathrm{m}), 7.817(1 \mathrm{H}, \mathrm{s})$, $8.729(1 \mathrm{H}, \mathrm{s}), 8.858(1 \mathrm{H}, \mathrm{s})$. IR $\left(\mathrm{KBr}, \mathrm{v}_{\max }, \mathrm{cm}^{-1}\right): 3054,2962$, 2924, 2853, 1710, 1619, 1551, 1464, 1395, 1262, 1217, 1097 , 1022, 867, 801, 694, 621. HR-MS (ESI): calcd. for $\mathrm{C}_{44} \mathrm{H}_{58} \mathrm{~N}_{3} \mathrm{O}_{8}$ $[\mathrm{M}+\mathrm{H}]^{+}:$756.4224. Found: 756.4200 .

6-Decyloxy-4-\{6-[2-(2-methoxy-1-methoxycarbonylvinyl)-phenoxy]-pyrimidin-4-yloxy\}-7-methyl-quinoline-3- 


\begin{tabular}{|c|c|c|c|c|c|c|}
\hline \multicolumn{7}{|c|}{$\begin{array}{l}\text { TABLE-1 } \\
\text { DATA FOR ANTICOCCIDIAL ACTIVITIES OF COMPOUNDS 7a-7h AND THE } \\
\text { COMPOUND DECOQUINATE AGAINST Eimeria tenella AT } 27 \text { mg/Kg }\end{array}$} \\
\hline Test groups & $\begin{array}{l}\text { Test compounds } \\
(\mathrm{mg} / \mathrm{Kg})\end{array}$ & $\begin{array}{c}\text { Rate of relative } \\
\text { body weight gain }\end{array}$ & $\begin{array}{l}\text { Survival } \\
\text { rate }(\%)\end{array}$ & $\begin{array}{l}\text { Lesion } \\
\text { scores }\end{array}$ & $\begin{array}{l}\text { Oocyst } \\
\text { scores }\end{array}$ & $\mathrm{ACI}^{\mathrm{a}}$ \\
\hline 1 & $7 \mathbf{a}(27)$ & 75.8 & 85 & 30 & 20 & 110.8 \\
\hline 2 & $7 b(27)$ & 70.3 & 85 & 30 & 20 & 105.3 \\
\hline 3 & $7 \mathbf{c}(27)$ & 87.7 & 90 & 20 & 20 & 137.7 \\
\hline 4 & 7d (27) & 86.7 & 90 & 20 & 20 & 137.7 \\
\hline 5 & $7 e(27)$ & 88.7 & 100 & 10 & 10 & 168.7 \\
\hline 6 & $7 f(27)$ & 72.8 & 85 & 30 & 20 & 107.8 \\
\hline 7 & $7 \mathrm{~g}(27)$ & 73.4 & 90 & 20 & 20 & 123.4 \\
\hline 8 & $7 \mathbf{h}(27)$ & 73.8 & 85 & 20 & 20 & 108.8 \\
\hline 9 & Decoquinate (27) & 91.3 & 100 & 10 & 1 & 180.3 \\
\hline 10 & $\mathrm{ING}^{\mathrm{b}}$ & 60.0 & 80 & 30 & 30 & 80 \\
\hline 11 & $\mathrm{NNG}^{\mathrm{c}}$ & 100.0 & 100 & 0 & 0 & 200 \\
\hline
\end{tabular}

${ }^{a}$ Anticoccidial activity index; ${ }^{b}$ Infected non-medicated group; ${ }^{\mathrm{c}}$ Non-infected non-medicated group

carboxylic acid ethyl ester (7c): Light yellow solid; yield: $42 \%$; m.p. 132-134 ${ }^{\circ} \mathrm{C}$; ${ }^{1} \mathrm{H}$ NMR spectrum $(400 \mathrm{MHz}$; $\mathrm{d}_{1}-\mathrm{CDCl}_{3}$; TMS): $\delta(\mathrm{ppm})=0.884(3 \mathrm{H}, \mathrm{t}, J=6.4 \mathrm{~Hz}), 1.278$ $(16 \mathrm{H}, \mathrm{m}), 1.838(3 \mathrm{H}, \mathrm{t}, J=6.4 \mathrm{~Hz}), 2.306(3 \mathrm{H}, \mathrm{s}), 3.617(3 \mathrm{H}$, s), $3.782(3 \mathrm{H}, \mathrm{s}), 4.107(2 \mathrm{H}, \mathrm{t}, J=6.8 \mathrm{~Hz}), 4.408(2 \mathrm{H}, \mathrm{q}, J=$ $6.8 \mathrm{~Hz}), 6.999(1 \mathrm{H}, \mathrm{s}), 7.26-7.52(6 \mathrm{H}, \mathrm{m}), 7.824(1 \mathrm{H}, \mathrm{s}), 8.723$ $(1 \mathrm{H}, \mathrm{s}), 8.866(1 \mathrm{H}, \mathrm{s})$. IR $\left(\mathrm{KBr}, \mathrm{v}_{\max }, \mathrm{cm}^{-1}\right): 3054,2958,2925$, 2853, 1706, 1618, 1550, 1449, 1395, 1262, 1217, 1096, 1023, 942, 874, 801, 687, 614, 551. HR-MS (ESI): calcd. for $\mathrm{C}_{38} \mathrm{H}_{46} \mathrm{~N}_{3} \mathrm{O}_{8}[\mathrm{M}+\mathrm{H}]^{+}:$672.3285. Found: 672.3264.

4-\{6-[2-(2-Methoxy-1-methoxycarbonyl-vinyl)-phenoxy]pyrimidin-4-yloxy \}-7-methyl-6-octyloxy-quinoline-3carboxylic acid ethyl ester (7d) : Light yellow solid; yield: $45 \%$; m.p. $130-132{ }^{\circ} \mathrm{C} ;{ }^{1} \mathrm{H}$ NMR spectrum (400 MHz; $\mathrm{d}_{1}-$ $\mathrm{CDCl}_{3}$; TMS): $\delta$ (ppm.) $=0.886(3 \mathrm{H}, \mathrm{t}, J=6.4 \mathrm{~Hz}), 1.248$ $(12 \mathrm{H}, \mathrm{m}), 1.833(3 \mathrm{H}, \mathrm{t}, J=6.8 \mathrm{~Hz}), 2.297(3 \mathrm{H}, \mathrm{s}), 3.608(3 \mathrm{H}$, s), $3.772(3 \mathrm{H}, \mathrm{s}), 4.100(2 \mathrm{H}, \mathrm{t}, J=6.8 \mathrm{~Hz}), 4.398(2 \mathrm{H}, \mathrm{q}, J=$ $6.4 \mathrm{~Hz}), 6.955(1 \mathrm{H}, \mathrm{s}) 7.22-7.44(6 \mathrm{H}, \mathrm{m}), 7.817(1 \mathrm{H}, \mathrm{s}), 8.712$ $(1 \mathrm{H}, \mathrm{s}), 8.858(1 \mathrm{H}, \mathrm{s}) . \mathrm{IR}\left(\mathrm{KBr}, \mathrm{v}_{\max }, \mathrm{cm}^{-1}\right) 3052,2963,2926$, 2855, 1707, 1617, 1551, 1449, 1398, 1262, 1217, 1022, 866, 801, 692, 613. HR-MS (ESI): calcd. for $\mathrm{C}_{36} \mathrm{H}_{42} \mathrm{~N}_{3} \mathrm{O}_{8}[\mathrm{M}+\mathrm{H}]^{+}$: 644.2972. Found: 644.2950.

6-Decyloxy-7-ethoxy-4-\{6-[2-(2-methoxy-1-methoxycarbonyl-vinyl)-phenoxy]-pyrimidin-4-yloxy\}-quinoline-3carboxylic acid ethyl ester (7e) : Light yellow solid; yield: 40 $\%$; m.p. 140-142 ${ }^{\circ} \mathrm{C} ;{ }^{1} \mathrm{H}$ NMR spectrum (400 MHz; $\mathrm{d}_{1}-\mathrm{CDCl}_{3}$; TMS): $\delta(\mathrm{ppm})=0.873(3 \mathrm{H}, \mathrm{t}, J=7.2 \mathrm{~Hz}), 1.264(16 \mathrm{H}, \mathrm{m})$, $1.407(3 \mathrm{H}, \mathrm{t}, J=6.8 \mathrm{~Hz}), 1.450(3 \mathrm{H}, \mathrm{t}, J=6.8 \mathrm{~Hz}), 3.573(3 \mathrm{H}$, s), $3.761(3 \mathrm{H}, \mathrm{s}), 3.961(2 \mathrm{H}, \mathrm{q}, J=6.8 \mathrm{~Hz}), 4.124(2 \mathrm{H}, \mathrm{t}, J=$ $6.4 \mathrm{~Hz}), 4.396(2 \mathrm{H}, \mathrm{q}, J=7.2 \mathrm{~Hz}), 6.875(1 \mathrm{H}, \mathrm{s}), 6.994(1 \mathrm{H}, \mathrm{s})$ $7.249(1 \mathrm{H}, \mathrm{s}) 7.32-7.47(4 \mathrm{H}, \mathrm{m}), 7.863(1 \mathrm{H}, \mathrm{s}), 8.706(1 \mathrm{H}, \mathrm{s})$, $8.863(1 \mathrm{H}, \mathrm{s})$. IR (KBr, $\left.v_{\max }, \mathrm{cm}^{-1}\right)$ 3054, 2927, 2854, 1707, 1620, 1553, 1507, 1451, 1382, 1270, 1226, 1131, 1097, 1025, 992, 952, 877, 801, 723, 691, 621, 592, 560. HR-MS (ESI): calcd. for $\mathrm{C}_{39} \mathrm{H}_{47} \mathrm{~N}_{3} \mathrm{O}_{9} \mathrm{Na}[\mathrm{M}+\mathrm{Na}]^{+}$: 724.3216. Found: 724.3210.

6-Dodecyloxy-4-\{6-[2-(2-methoxy-1-methoxycarbonyl-vinyl)-phenoxy]-pyrimidin-4-yloxy\}-7-methylquinoline-3-carboxylic acid ethyl ester (7f) : Light yellow solid; yield: $42 \%$; m.p. $135-136{ }^{\circ} \mathrm{C}$; ${ }^{1} \mathrm{H}$ NMR spectrum $(400$ $\mathrm{MHz} ; \mathrm{d}_{1}-\mathrm{CDCl}_{3}$; TMS $): \delta(\mathrm{ppm})=0.881(3 \mathrm{H}, \mathrm{t}, J=6.4 \mathrm{~Hz})$, $1.228(20 \mathrm{H}, \mathrm{m}), 1.838(3 \mathrm{H}, \mathrm{t}, J=6.8 \mathrm{~Hz}), 2.303(3 \mathrm{H}, \mathrm{s}), 3.616$ $(3 \mathrm{H}, \mathrm{s}), 3.779(3 \mathrm{H}, \mathrm{s}), 4.105(2 \mathrm{H}, \mathrm{t}, J=6.4 \mathrm{~Hz}), 4.406(2 \mathrm{H}, \mathrm{q}$,
$J=6.8 \mathrm{~Hz}), 6.964(1 \mathrm{H}, \mathrm{s}) 7.22-7.45(5 \mathrm{H}, \mathrm{m}), 7.489(1 \mathrm{H}, \mathrm{s})$, $7.823(1 \mathrm{H}, \mathrm{s}), 8.718(1 \mathrm{H}, \mathrm{s}), 8.864(1 \mathrm{H}, \mathrm{s})$. IR $\left(\mathrm{KBr}, \mathrm{v}_{\max }, \mathrm{cm}^{-1}\right)$ $3055,2926,2853,1706,1618,1551,1450,1395,1267,1218$, 1131, 1098, 1025, 992, 943, 881, 802, 764, 723, 688, 615. HR-MS (ESI): calcd. for $\mathrm{C}_{40} \mathrm{H}_{50} \mathrm{~N}_{3} \mathrm{O}_{8}[\mathrm{M}+\mathrm{H}]^{+}: 700.3598$. Found: 700.3573 .

6-Benzyloxy-4-\{6-[2-(2-methoxy-1-methoxycarbonyl-vinyl)-phenoxy]-pyrimidin-4-yloxy\}-7-methylquinoline-3-carboxylic acid ethyl ester (7g): Light yellow solid; yield: $40 \%$; m.p. $156-158{ }^{\circ} \mathrm{C}$; ${ }^{1} \mathrm{H}$ NMR spectrum $\left(400 \mathrm{MHz} ; \mathrm{d}_{1}-\mathrm{CDCl}_{3} ; \mathrm{TMS}\right): \delta(\mathrm{ppm})=1.424(3 \mathrm{H}, \mathrm{t}, J=$ $7.2 \mathrm{~Hz}), 2.374(3 \mathrm{H}, \mathrm{s}), 3.621(3 \mathrm{H}, \mathrm{s}), 3.788(3 \mathrm{H}, \mathrm{s}), 4.419$ $(2 \mathrm{H}, \mathrm{t}, J=7.2 \mathrm{~Hz}), 4.402(2 \mathrm{H}, \mathrm{q}, J=6.8 \mathrm{~Hz}), 5.231(2 \mathrm{H}, \mathrm{s})$, $7.000(1 \mathrm{H}, \mathrm{s}), 7.22-7.54(11 \mathrm{H}, \mathrm{m}), 7.950(1 \mathrm{H}, \mathrm{s}), 8.752$ $(1 \mathrm{H}, \mathrm{s}), 8.870(1 \mathrm{H}, \mathrm{s})$. IR $\left(\mathrm{KBr}, \mathrm{v}_{\max }, \mathrm{cm}^{-1}\right) 3060,2936,1732$, $1707,1627,1558,1489,1447,1384,1252,1213,1168$, 1134, 1101, 1021, 946, 855, 804, 758, 699, 622, 588, 461. HR-MS (ESI): calcd. for $\mathrm{C}_{35} \mathrm{H}_{31} \mathrm{~N}_{3} \mathrm{O}_{8} \mathrm{Na}[\mathrm{M}+\mathrm{Na}]^{+}$: 644.2009 . Found:644.2009.

6-Benzyloxy-7-methoxy-4-\{6-[2-(2-methoxy-1methoxycarbonyl-vinyl)-phenoxy]-pyrimidin-4-yloxy\}quinoline-3-carboxylic acid ethyl ester (7h): Light yellow solid; yield: $40 \%$; m.p. $184-187^{\circ} \mathrm{C} ;{ }^{1} \mathrm{H}$ NMR spectrum (400 $\mathrm{MHz} ; \mathrm{d}_{1}-\mathrm{CDCl}_{3}$; TMS): $\delta(\mathrm{ppm})=1.412(3 \mathrm{H}, \mathrm{t}, J=6.4 \mathrm{~Hz})$, $3.573(3 \mathrm{H}, \mathrm{s}), 3.778$ (3H, s), 3.818 (3H, s), 4.410 (2H, q, J=7.2 $\mathrm{Hz}), 5.265(2 \mathrm{H}, \mathrm{s}), 6.911(1 \mathrm{H}, \mathrm{s}), 7.059(1 \mathrm{H}, \mathrm{s}), 7.22-7.50(10 \mathrm{H}$, m), $7.964(1 \mathrm{H}, \mathrm{s}), 8.733(1 \mathrm{H}, \mathrm{s}), 8.874(1 \mathrm{H}, \mathrm{s})$. IR (KBr, $\mathrm{v}_{\max }$, $\left.\mathrm{cm}^{-1}\right) 3059,2952,1621,1586,1551,1450,1387,1265,1218$, 1132, 1098, 1024, 944, 857, 802, 696, 462. HR-MS (ESI): calcd. for $\mathrm{C}_{35} \mathrm{H}_{31} \mathrm{~N}_{3} \mathrm{O}_{9} \mathrm{Na}[\mathrm{M}+\mathrm{Na}]^{+}$: 660.1958. Found: 660.1959.

Biological assay: The anticoccidial activities of $\mathbf{7 a - 7 h}$ were evaluated according to the anticoccidial index method, using decoquinate as a reference drug ${ }^{15,16}$. The chickens used to test the anticoccidial activity of compounds were 15-dayold broiler chickens, which fed by the feedstuff without any anticoccidial drugs and drank clean water by Guangdong province academy of agricultural sciences. Then these chickens were randomly divided into 11 groups, 20 in each group. Groups 1-9 of these chickens were fed the diet with the compounds $\mathbf{7 a - 7 h}$ or decoquinate in $27 \mathrm{mg} / \mathrm{Kg}$. Groups $1-10$ of these chickens were infected factitiously with the Eimeria tenella. After 7 days, recorded the weight gain, mortality, 
dropping scores, lesion scores and oocysts scores of the chickens to calculate the anticoccidial index.

\section{RESULTS AND DISCUSSION}

Synthesis: The previous steps were almost synthesized according to the literatures, the final and most important step is to synthesize target compounds. Primarily $\mathrm{NaH}$, quinoline carboxylates and intermediate 6 were stirred at $90{ }^{\circ} \mathrm{C}$ for $5 \mathrm{~h}$ in order to give the target compounds, but formed lots of by-products and the purification process was very difficult. Subsequently, $\mathrm{K}_{2} \mathrm{CO}_{3}$ was used in place of $\mathrm{NaH}$ to get target compounds, formed little by-products and the purification process become easy.

Temperature was another principal influencing element. The synthetic reaction of $\mathbf{7} \mathbf{a}-\mathbf{h}$ ran slowly at room temperature. The elevation of temperature could accelerate the process, 90 95 ${ }^{\circ} \mathrm{C}$ was the optimal condition.

The amount of intermediate $\mathbf{6}$ should be excessive to intermediate $\mathbf{5}$, because intermediate $\mathbf{5}$ was difficult to scrub out. The ratio of intermediate $\mathbf{5 a - 5 h}: \mathrm{K}_{2} \mathrm{CO}_{3}$ : intermediate $\mathbf{6}$ was set at 1:1.3:1.2.

In this reaction, we found that the reaction time could be shortened by adding KI as a catalyze.

Biological activity: The results of anticoccidial activities of the compounds $\mathbf{7 a - 7 h}$ are listed in Table-1. The results showed that four of the target compounds exhibited good anticoccidial activities with a dose of $27 \mathrm{mg} / \mathrm{Kg}$. Among them, compound 7e showed strong anticoccidial activity with anticoccidial index 168.7, might be developed as available anticoccidial drugs. Meanwhile compounds 7c, 7d and 7g exhibited obvious anticoccidial activities, with anticoccidial index 137.7, 137.7 and 123.4.

\section{Conclusion}

A series of novel quinoline carboxylate derivatives with methyl(E)-2-(3-methoxy) acrylate group were designed and synthesized as anticoccidial medicines. The anticoccidial activity results indicated that compound 7e showed a high anticoccidial activityat a dose of $27 \mathrm{mg} / \mathrm{Kg}$, might be a new anticoccidial drug.

\section{ACKNOWLEDGEMENTS}

The authors thank for the financial support from the National Science Foundation of China ((No. 21072135) and ${ }^{1} \mathrm{H}$ NMR analysis by Sichuan University Analytical \& Testing Center. The authors appreciated the anticoccidial activity test by South China Agricultural University.

\section{REFERENCES}

1. R.B. Williams, Int. J. Parasitol, 29, 1209 (1999).

2. C.F. Duffy, G.F. Mathis and R.F. Power, Vet. Parasitol., 130, 185 (2005).

3. D. Feng, M. Fisher, G.B. Liang, X.X. Qian, C. Brown, A. Gurnett, P.S. Leavitt, P.A. Liberator, J. Mathew, A. Misura, S. Samaras, T. Tamas, D.M. Schmatz, M. Wyvratt and T. Biftu, Bioorg. Med. Chem. Lett., 16, 5978 (2006).

4. H.D. Chapman, Avian. Pathol., 26, 221 (1997).

5. G. Greif and B. Stephan, Parasitol. Res., 82, 706 (1996).

6. J.J. Xu, J.P. Tao and J.B. Peng, Anim. Husbandry Vet. Med., 40, 18 (2008).

7. Y.Y. Zhang and Y.L. Wang, Chinese Chem. Lett., 21, 426 (2010).

8. J.N. Hodgson, Br. Vet. J., 124, 209 (1968).

9. G.Q. Li, S. Kanu, F.Y. Xiang, S.M. Xiao, L. Zhang, H.W. Chen and H.J. Ye, Vet. Parasitol., 119, 261 (2004).

10. J.H. You, C.W. Ye, Y.B. Weng, X.H. Mo and Y.L. Wang, Arkivoc., 1 (2008).

11. C.R. Yan, J. Xu, Y.B. Weng, J. Li, Y.L. Wang and H. Chen, Chem. Biol. Drug. Des., 72, 314 (2008).

12. J. Xu, C.R. Yan, Y.B. Weng, L.L. Qiu and Y.L. Wang, Chem. Res. Appl., 21, 694 (2009).

13. J. Dong, H. Dao, J.S. Lou and H.J. Pi, Fine Chem. Intermed., 37, 25 (2007).

14. C.R. Yan, L. Li, K.Q. Wu and Y.L. Wang, J. Yibin Uni., 5 (2012).

15. J.J. Xu, J.P. Tao and J.B. Peng, Anim. Husbandry Vet. Med., 40, 18 (2008).

16. S.D. Folz, B.L. Lee and L.H. Nowakowski, Int. J. Parasitol., 75, 696 (1989). 